\title{
The race to wire up the poor
}

Nicholas Negroponte's much hyped 'One Laptop Per Child' (OLPC) scheme inspires or infuriates according to taste. The idea of revolutionizing education by distributing millions of '\$100 laptops' to children in the world's poorest countries can be seen as Soviet-style social engineering or as visionary California dreaming, mass empowerment or pointless frivolity. However you see it, though, the project is facing real problems - and competition.

Negroponte, who founded the MIT Media Lab in Cambridge, Massachusetts, and heads the non-profit OLPC Foundation, admitted last week that in the near term the laptops will cost not US $\$ 100$, but at least $\$ 175$. And although he is still confident that the first 3 million machines will be shipped by the end of 2007, an earlier estimate for the same timeframe was 10 million.

Meanwhile, as PC markets in industrialized nations become saturated, Intel, Microsoft, and other computer companies are turning their sights on developing countries, hoping to find the next billion computer users.

"We are at the most critical stage of OLPC's life," Negroponte told a meeting with industry analysts in Cambridge, Massachusetts, on 26 April. "A year and a half ago, we were selling a dream, but it's easy to sell dreams if you're passionate and can share that passion with other people. But that was dreams, and now we have got to launch."

\section{Unique approach}

To launch, Negroponte needs money. OLPC has an unorthodox business model: it sells only to governments and demands minimum orders of 250,000 machines at a time - down from its original demand of one million units. The idea is to achieve scale instantaneously, and to pass distribution and marketing costs on to the purchaser.

How much those extra costs will push the bill up is not clear. The OLPC project is based on the idea that just providing computers can make a huge difference, as children will spontaneously use them to make up for what they lack in books and other equipment and experiences. But many education researchers dispute this theory. And the more that classroom structure and teacher training is needed to maximize the computers' benefit, the more the programme will end up costing governments.

So far no countries have actually paid for their computers, let alone revamped their education efforts to prepare. Libya has come closest, signing a memorandum of under-

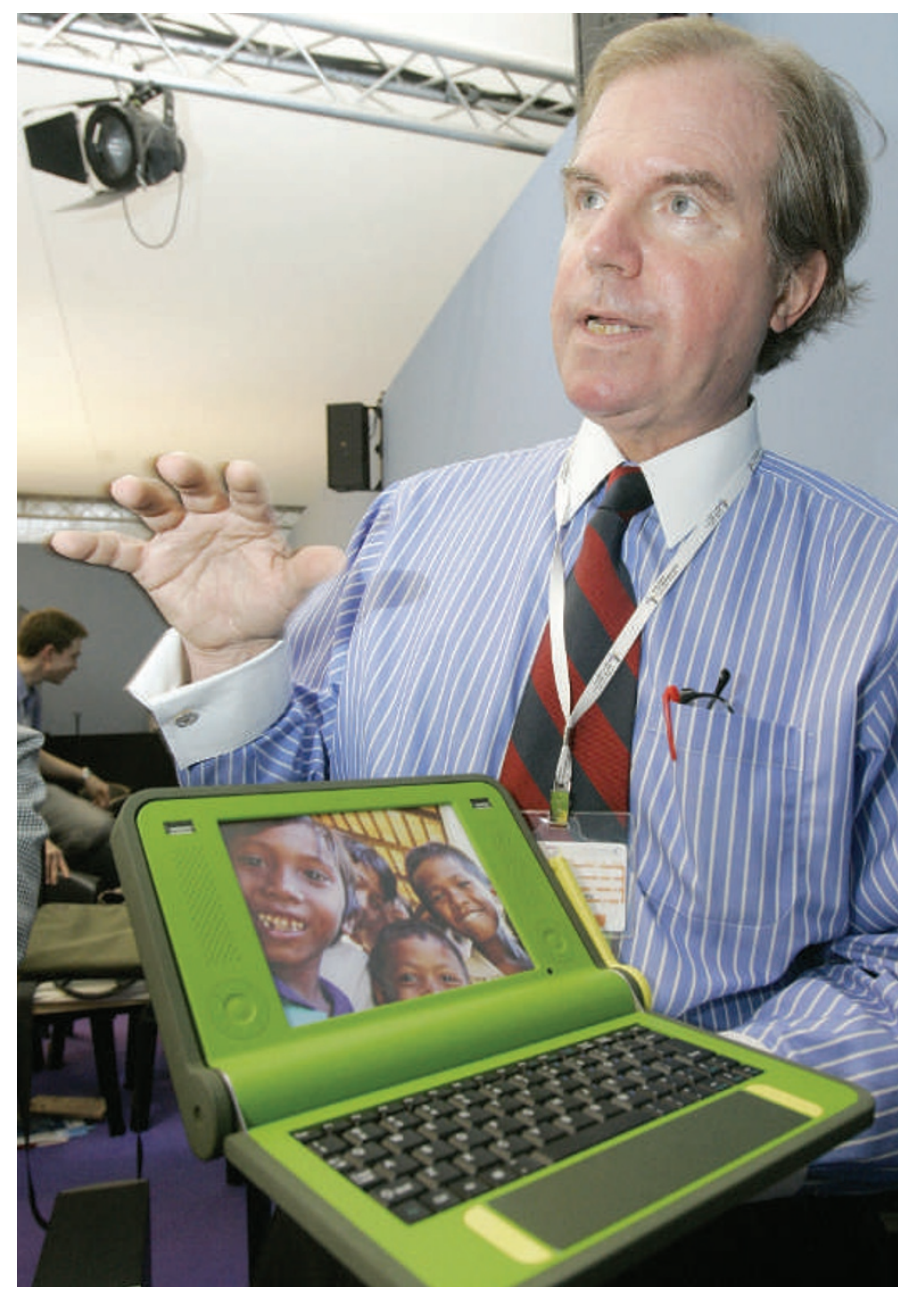

Nicholas Negroponte hopes to get computers to children in developing countries though his One Laptop Per Child scheme. standing to buy 1.2 million laptops. Rwanda and Uruguay have said that they are committed to the project, and several other countries have expressed interest. But some are firmly ruling themselves out. "We cannot visualize a situation for decades when we can go beyond the pilot stage," Sudeep Banerjee, India's education secretary, said last year. "We need classrooms and teachers more urgently than fancy tools."

In March, in an investment report on OLPC and related companies such as Quanta, the Taiwanese firm contracted to build the foundation's \$100 laptop - investment analysts at Merrill Lynch concluded that "despite the hype, we urge investors not to chase stocks related to OLPC". Low-end laptops can already be made at a cost of $\$ 400$, the report pointed out, and OLPC's lower costs come from using less-powerful components and eliminating

distribution and after-sales service, both of which impose burdens on the end user.

The report also points out that OLPC is not the only show in town. Intel is rolling out its $\$ 400$ Classmate PC to schools in Brazil and Mexico this year, and has pilot schemes in 25 other countries. Over the next 5 years it

"We need classrooms and teachers more urgently than fancy tools." is investing $\$ 1$ billion to promote access to information technology in developing countries. Microsoft, loathe to see the next billion computer users running the open-source Linux operating system that the OLPC computers use, last week announced that it would offer developing countries a software package that includes XP and Office for $\$ 3$ for every new school PC bought.

No one denies that the rugged OLPC laptop contains some serious technological innovation. It runs on just 2 watts of electric- 
ity - around a twentieth of a typical laptop's needs - and can be charged off-grid in various ways. Its display can be read in bright sunlight, and nearby laptops automatically create a wireless network between themselves with which, among other things, they can share an Internet connection.

Ashok Jhunjhunwala, a former director of the Indian Institute of Technology in Chennai, and one of the India's top experts in information technology sees the OLPC machine as "a great technological effort". But "proper support is a must. Nothing works without support, management and maintenance".

\section{Change in focus}

Poor countries have other public spending priorities, Jhunjhunwala argues, and initiatives such as OLPC should start where they can have a real effect - among the market of emerging middle classes and schools that can afford the computers for themselves. The OLPC will otherwise be irrelevant to developing countries; in the poorest strata of society "this toy will just be sold or stolen".

Jhunjhunwala himself is the brains behind the netPC and netTV computers built by Novatium, a Chennai-based company, which embraces the idea that PCs no longer need large, expensive hard drives and stacks of memory, but can instead act as gateways to computing power elsewhere on the Internet. Users are expected to access free web-based word-processing and spreadsheet software and storage, as offered by web services such as Google Office, or to take out a subscription for Microsoft Office and other paying software served over the web by Novartium's own central servers.

Whatever the future for the OLPC project, the fact that the next billion computer users will be in developing countries looks set to drive a new phase in computing innovation - one that could have repercussions in developed markets too. Last week, for the first time, Negroponte said that he was considering selling the computers to US children via their state governments, an idea he had previously rejected. The educational information appliance of the future might turn out to be a mobile telephone, or some sort of hybrid, but no one can rule out Negroponte's vision of one laptop per child coming true worldwide. That does not mean, though, that his specific approach will get much further.

Declan Butler

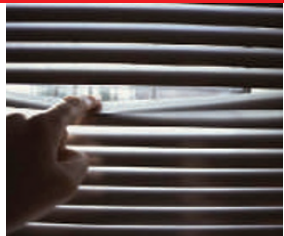

QUANTUM CRYPTOGRAPHY IS HACKED

It is possible to eavesdrop on encrypted messages. www.nature.com/news

\section{Long odds on a long shadow}

Astronomers may make a major step forward in studying planets around other stars as early as next week - but the odds are strongly against them.

Last week's announcement by Stéphane Udry and his colleagues at the Geneva Observatory that they had found a planet in the 'habitable zone' of the red dwarf Gliese 581 caused a great deal of excitement (S. Udry et al. Astron. Astrophys.; in the press). Its low mass (about five times that of Earth) and its position in the habitable zone, where temperatures are compatible with water being present as a liquid, mean that Gliese 581 c, as the planet is called, could be more Earth-like than anything else yet seen beyond the Solar System.

However, finding out whether Gliese $581 \mathrm{c}$ is a larger version of Earth or a smaller version of Neptune - a much less homely prospect - means measuring its density. The only way this can be done is by observing the planet cross the face of Gliese 581 and thus working out its radius. Unfortunately, such a transit will be visible from our Solar System only if we happen to be sitting in the plane of the planet's orbit. The chances of that being the case are a daunting 50 to 1 against. Nevertheless, any odds are worth taking when it's the only game in town. On 26 April, just three days after the planet's existence was revealed, Dimitar Sasselov of Harvard University began using the small Canadian space telescope MOST (Microvariability and Oscillations of Stars) to observe Gliese 581. Sasselov predicts that, if the geometry is right, his team should see the star dim on 7 May as the planet passes in front of it.

"If Gliese 581 c transits then the doors are open" says David Charbonneau, another Harvard exoplanet hunter. A transit would not only supply data about density, it would open up the possibility of follow-on observations that might reveal clues to the contents of the planet's atmosphere. A definitive failure to see a transit, on the other hand, would close off almost all lines of future observation with current technologies. An exception is listening out for evidence of artificial radio transmissions. The SETI Institute of Mountain View, California, kept an ear out for signals from Gliese 581 in 1995 and 1997. "We didn't find any signal," says Seth Shostak, a SETI Institute astronomer. But the institute's next project - the Allen telescope - will nevertheless make looking at the Gliese 581 system a priority.

Without transits, the wild card of SETI is the only way forward on this particular planet. But Charbonneau is He draws the inference from Gliese 581 c that there are more such low-mass planets around dwarf stars, and indeed Udry says he already has candidates for which he needs just a bit more data. It's not unreasonable to hope that within a few years enough data will have been found to make it likely that at least one will be observable in transit.

"One day we'll be lucky,"

Sasselov agrees. "And hopefully," he adds, "it'll be next week."

Katharine Sanderson still uplifted by the discovery.

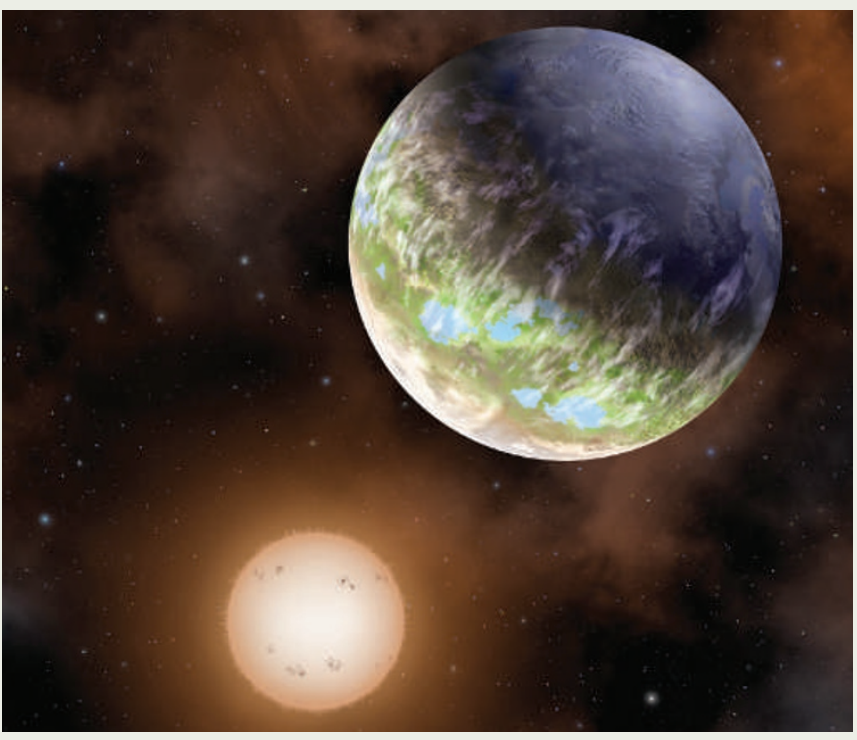

Planets in the habitable zones of red dwarfs could be strange new Earths. 